\title{
Downregulation of NONO induces apoptosis, suppressing growth and invasion in esophageal squamous cell carcinoma
}

\author{
RUI CHENG ${ }^{1-4}$, SHENGTAO ZHU ${ }^{1-4}$, SHUILONG GUO ${ }^{1-4}$, LI MIN $^{1-4}$, JIE XING $^{1-4}$, \\ QINGDONG GUO ${ }^{1-4}$, PENG LI ${ }^{1-4}$ and SHUTIAN ZHANG ${ }^{1-4}$ \\ ${ }^{1}$ Department of Gastroenterology, Beijing Friendship Hospital, Capital Medical University; \\ ${ }^{2}$ National Clinical Research Center for Digestive Diseases; ${ }^{3}$ Beijing Digestive Disease Center; \\ ${ }^{4}$ Beijing Key Laboratory for Precancerous Lesion of Digestive Diseases, Beijing 100050, P.R. China
}

Received June 5, 2017; Accepted December 1, 2017

DOI: 10.3892/or.2018.6334

\begin{abstract}
Esophageal squamous cell carcinoma (ESCC) is one of the most common malignancies in China, and is associated with high morbidity and mortality. However, the molecular mechanisms that control ESCC tumorigenicity and metastasis remain unclear. Here, we report that the RNA splicing factor, NONO, is an important regulator of ESCC growth, apoptosis and invasion. NONO protein levels were dramatically upregulated in ESCC when compared with that in adjacent benign esophageal squamous epithelium. Particularly, NONO expression was statistically higher in tumors with greater tumor invasion depth. Using multiple ESCC cell models, we further showed that NONO depletion using siRNA significantly inhibited proliferation, invasion, and promoted apoptosis of ESCC cells. In addition we found that knockdown of NONO could reduce protein levels of phosphorylated Akt and Erk1/2. Our findings suggest that NONO plays a potent role in multiple biological aspects of ESCC through activation of the Akt and Erk1/2 signaling pathways. Taken together, our findings suggest that NONO might play an important role in promoting tumorigenesis of ESCC. It may provide a promising approach to prevent the progress of ESCC.
\end{abstract}

\section{Introduction}

Esophageal cancer is one of the most aggressive cancers worldwide. There are two main types of esophageal cancer: esophageal squamous cell carcinoma (ESCC) and esophageal

Correspondence to: Dr Shutian Zhang, Department of Gastroenterology, Beijing Friendship Hospital, Capital Medical University, 95 Yong An Road, Xi Cheng, Beijing 100050, P.R. China

E-mail: zhangshutian@ccmu.edu.cn

Key words: NONO, esophageal squamous cell carcinoma, apoptosis, migration, invasion adenocarcinoma (EAC) (1). In Asia, ESCC is the predominant type and EAC remains rare (2). Treatment of ESCC has greatly relied upon classical therapeutic means including surgery, radiotherapy or chemotherapy. Although multimodality therapy can apparently improve the prognosis of patients with ESCC, the overall 5-year survival rate remains unsatisfactory. Therefore, seeking new potential targets for the therapy of ESCC will help to improve the clinical outcome of patients with ESCC (3), which is reflecting our limited understanding on carcinogenesis and metastasis of ESCC at the molecular and cellular levels. It is urgent to find sensitive and specific early biomarkers for diagnosis and prognosis of ESCC, as well as novel therapeutic strategies.

The non-POU-domain-containing octamer (NONO) binding protein, also called NONO, is involved in a variety of biological processes, including regulating gene expression, DNA synthesis and repair processes (4-6). Originally identified as an RNA-binding protein (7), NONO interacts with double-stranded DNA, single-stranded DNA and RNA and is involved in multiple steps of gene transcription, RNA splicing and even non-homologous DNA end joining (8). Due to its roles in gene transcription, RNA processing and DNA repairing, NONO may be implicated in cancer progression. It has been demonstrated that NONO is associated with the initiation and progression of human cancer. NONO protein expression is strongly correlated with vascular invasions and poor patient survival of bladder cancers (9). In fact, increased NONO expression was reported in prostate cancer and malignant melanoma $(10,11)$. Knockdown NONO reduced melanoma cell proliferation, promoted apoptosis and decreased mobility capacity. Overexpression of NONO increased human umbilical vein endothelial cells invasion (12). Chromosomal translocation of NONO and TFE3 genes are frequently detected in papillary rental cell carcinoma (13). Additionally, a previous study indicates that NONO is an independent prognostic factor for human neuroblastoma (14). One recent study has identified that the nuclear protein NONO is highly expressed in breast cancer tissues as compared with the adjacent normal tissues in human patients. It is also demonstrated that NONO-mediated regulation of breast cancer cell growth is dependent on the 
presence of and interaction with SREBP-1 (15). Moreover, gene microarray studies also reported that NONO mRNA levels are increased in ESCC $(16,17)$. However, validation of NONO protein expression in human ESCC tissues has not been performed. The protein expression and functional significance of NONO in ESCC are still unclear.

\section{Materials and methods}

Ethical approval. All procedures performed in studies involving human participants were in accordance with the ethical standards of the institutional and/or national research committee and with the 1964 Helsinki declaration and its later amendments or comparable ethical standards. Collection of all tissues and data involving human participants were approved by institutional ethics committee of Beijing Friendship Hospital on human research.

Human tissue specimens and cell lines. Human ESCC and adjacent normal esophageal tissues were collected from 42 patients undergoing surgery of ESCC at the Beijing Friendship Hospital (Beijing, China) between 2008 and 2012. No patients had been treated with chemo- or radiation therapy before surgery. Informed consent was obtained from each patient. All tissues and data were collected in accordance with institutional guidelines and approved by institutional ethics committee of Beijing Friendship Hospital on human research. The pathological tumor stage was assessed using the TNM system according to the American Joint Committee on Cancer (AJCC) staging manual (seventh edition) $(18,19)$. Human ESCC cell lines (TE-1, TE-2, TE-10, KYSE30, KYSE70, KYSE140, KYSE450, EC109, EC9706) were generously provided by Cancer Institute and Hospital, Chinese Academy of Medical Sciences and cell authentication were reported $(20,21)$. Cells were maintained in RPMI-1640 medium containing $10 \%$ fetal bovine serum, at $37^{\circ} \mathrm{C}$ in a $95 \%$ humidified incubator containing $5 \% \mathrm{CO}_{2}$.

Immunohistochemistry and pathological scoring. Immunohistochemistry (IHC) followed the standard protocol with NONO antibody (BD Biosciences, Bedford, MA, USA). Slides were also counterstained with hematoxylin and mounted on coverslips with glycerol-gelatin. Pathological scoring of NONO was performed by the quickscore method (22). The percentage of stained cells $(0-4,5-19,20-35,40-59,60-79$ and $80-100 \%$ ) was scored as $1-6$, while the staining intensity (no staining, low, moderate, and high) was scored as 0-3. The quickscore of each tissue sample was calculated by multiplying the score of percentage of positive cells with the score of the staining intensity. Tumor samples with quickscore $>12$ were recorded as strong, moderate (quickscore $<12$ and $>6$ ), weak positive/negative (quickscore $<6$ ). Two doctors with no prior knowledge of each patient's clinical information evaluated all specimens independently.

siRNA and transfection. TE-1 and KYSE70 cells were plated at a density of $4 \times 10^{5}$ cells/well in 6 -well plates. After $24 \mathrm{~h}$ of culture, the cells were transfected with siRNA using Lipofectamine ${ }^{\circledR} 2000$ (Life Technologies, Grand Island, NY, USA) according to the manufacturer's protocol as we previously described. Small interfering RNA (siRNA) for NONO (siNONO) and control siRNA (siCTRL) were purchased from GenePharma (Shanghai, China). The siRNA sequences against NONO are 5'-CAGAGA AGCUG GUUAUAAAT-3' and 5'-UUUAUAACCAGCUUCU CUGTT-3'. The siRNA sequences against non-specific are 5'-UUCUCCGAACGUGUCACGUTT-3' and 5'-ACGUGAC ACGUUCGGAGAATT-3'.

Real-time PCR. Total RNA was extracted from ESCC cell lines using TRIzol (Life Technologies) as we previously described (23). Two micrograms of total RNA was subjected to a random-primed reverse transcription using SuperScript II reverse transcriptase (Life Technologies). Real-time qPCR was conducted in triplicates using Applied Biosystems 7500 Fast with $5 \mathrm{ng}$ of cDNA, $1 \mu \mathrm{M}$ of each primer pair and SYBR Green PCR master mix (Roche). The primers used were NONO forward, 5'-TTGTGGGAAATCTTCCTCCCGACA-3' and reverse, 5'-GGGTTTCCAAGCGGATAAAGCCAA-3'. GAPDH forward, 5'-GGACCTGACCTGCCGTCTAGAA-3' and reverse, 5'-GGTGTCGCTGTTGAAGTCAGAG-3'. Relative mRNA levels were normalized to GAPDH.

Western blot analysis. Antibodies against Akt, phosphorylated Akt (p-Akt) (Ser473), Erk, p-Erk1/2 (Thr202/Tyr204), caspase-3 and cleaved PARP-1 were purchased from Cell Signaling Technology (Beverly, MA, USA). The antibody against $\beta$-actin was purchased from Sigma-Aldrich. Protein lysates were extracted by lysis buffer [50 mM Tris-HCl, pH 7.4; 10 mM EDTA; 5 mM EGTA; 0.5\% NP40; 1\% Triton X-100 plus protease inhibitor (Roche)]. Fifty micrograms of total protein were fractionated by SDS-polyacrylamide gel electrophoresis and then transferred to PVDF membranes (Millipore, Billerica, MA, USA). The membranes were probed with primary antibodies overnight at $4^{\circ} \mathrm{C}$ and then incubated for $1 \mathrm{~h}$ with secondary peroxidase-conjugated antibodies at room temperature, followed by detection with an ECL plus system (Beyotime, Jiangsu, China). $\beta$-actin was used as the loading control.

Cell proliferation assay. Cells were plated onto a 96-well plate at a density of $1 \times 10^{3}$ cells per well. Viability of the cells was measured using a CellTiter $96^{\circledR}$ Aqueous One Solution Cell Proliferation assay (Promega, Madison, WI, USA) as described previously with modifications (20). After 0, 24, 48 and $72 \mathrm{~h}$, the optical density at $492 \mathrm{~nm}$ was measured on a microplate reader (Smartspec Model 450; Bio-Rad Laboratories, Inc., Hercules, CA, USA) at $490 \mathrm{~nm}$. Three independent experiments were performed in triplicate.

Cell apoptosis analysis. Apoptosis rates of cells were measured using an Annexin V-FITC/PI apoptosis kit (Becton Dickinson, Franklin Lakes, NJ, USA) following the manufacturer's instructions. Briefly, cells were harvested, washed with cold PBS, and resuspended in binding buffer. Then, $5 \mu$ l Annexin V-FITC and $5 \mu \mathrm{l}$ PI were added to each sample containing $1 \times 10^{5}$ cells $/ 100 \mu 1$. The samples were incubated at $25^{\circ} \mathrm{C}$ in the dark for $15 \mathrm{~min}$, followed by addition of $400 \mu \mathrm{l}$ binding buffer. Within $1 \mathrm{~h}$ of preparation, the samples were analyzed by a flow cytometer (Becton Dickinson). The experiments were repeated three times independently. 

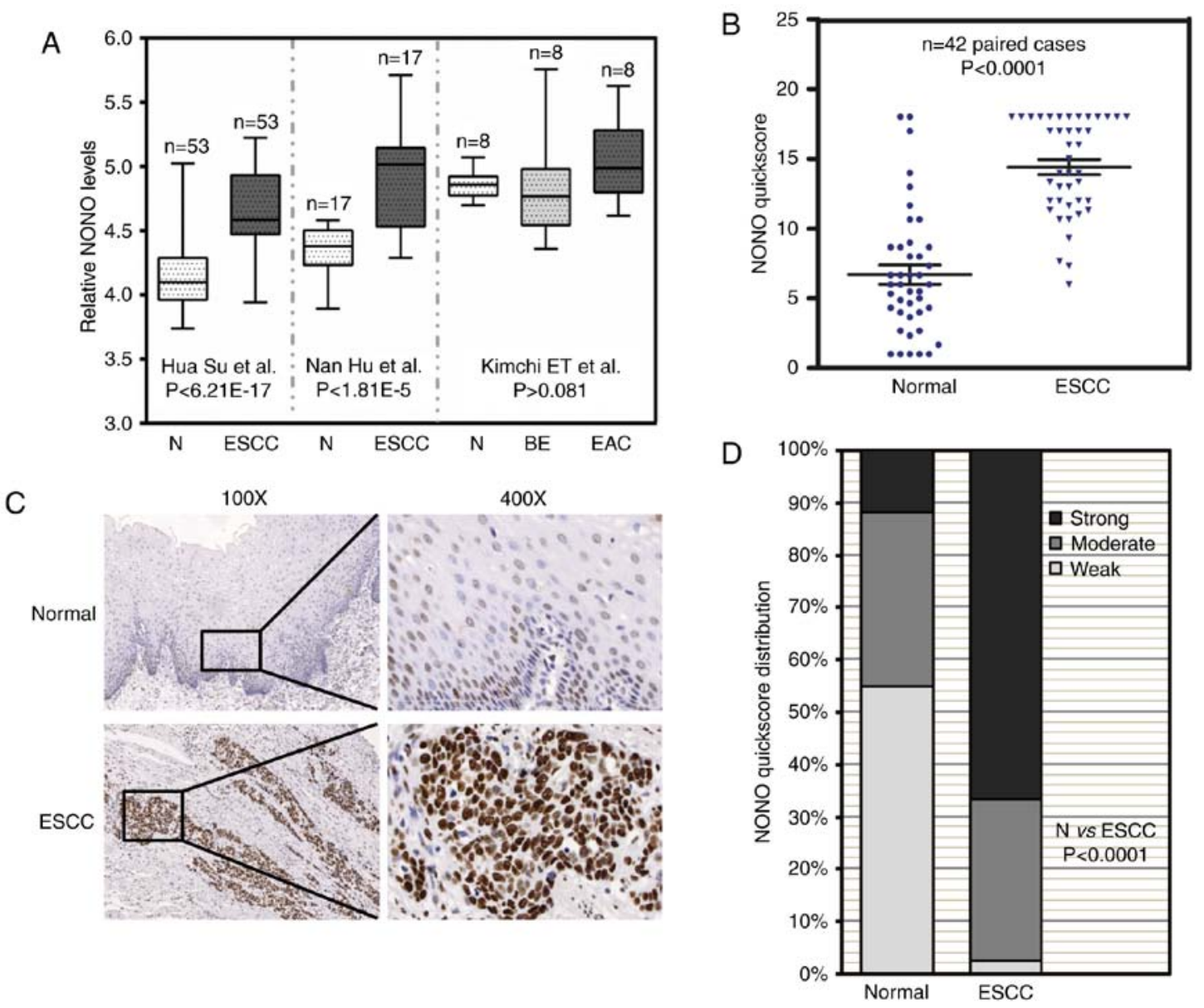

Figure 1. Increased expression of NONO in ESCC tissue samples. (A) Three independent microarray data from the Oncomine database showed that NONO mRNA levels were increased in ESCC patient tissue samples. Comparison of NONO mRNA levels in normal tissue (N), esophageal squamous cell carcinoma (ESCC), and Barrett's esophagus (BE), esophageal adenocarcinoma (EAC) are presented together with P-values. (B) Immunohistochemistry signal of NONO from paired normal and ESCC tissue samples were recorded by quickscore method. Comparison of quickscore distribution between adjacent normal and ESCC samples was performed by $\chi^{2}$ test. (C) Representative immunohistochemistry (IHC) images of NONO in paired normal and ESCC tissue samples. NONO levels were lower in adjacent normal esophageal epithelium, but were higher in ESCC. (D) NONO quickscores were further divided into three groups: strong, moderate and weak. The percentage of each group in normal and ESCC tissue samples were plotted.

Cell migration and invasion assays. In cell migration assays, cells were grown on 6 -well plates until $100 \%$ confluent. A 20- $\mu 1$ pipette tip was used to scratch and create a wound in the confluent monolayer at the center of culture plates. After washes with PBS buffer, cells were replenished with culture medium and cell migration was captured under a microscope (Olympus Corp., Tokyo, Japan) at 0 and $24 \mathrm{~h}$ post wound scratch. Migration rate was determined using the ImageJ software as an average closed area of the wound relative to the initial wound area at $24 \mathrm{~h}$ after wounding. Experiments were performed in triplicate with three independent repeats. In cell invasion assays, $10 \times 10^{4}$ of TE- 1 cells, $12 \times 10^{4}$ of KYSE70 cells were suspended in serum-free RPMI-1640 medium and seeded in BD Matrigel invasion chamber (Becton Dickinson). The lower chamber contained $0.75 \mathrm{ml}$ of RPMI-1640 medium plus $10 \%$ FBS. After incubation for $30 \mathrm{~h}$, non-invading cells in the upper chamber were gently removed by cotton swabs. Cells that invaded through the Matrigel and reached to the lower chamber were fixed, stained with mounting medium containing DAPI (Vector Laboratories, Inc., Burlingame, CA, USA) and photographed by fluorescence microscope (Olympus). Invaded cell numbers were counted by the ImageJ software. Experiments were performed in triplicate with three independent repeats.

Immunofluorescence assays. ESCC cell lines were grown on glass coverslips (Nest Biotechnology Co., Ltd., Wuxi, China) to $50 \%$ confluence and then fixed in $4 \%$ paraformaldehyde for $15 \mathrm{~min}$ at room temperature. The cells were permeabilized with $0.3 \%$ Triton X-100 for 20 min, blocked with $5 \%$ bovine serum albumin for $30 \mathrm{~min}$, and then incubated with the mouse monoclonal anti-NONO antibody (Becton Dickinson) at $4^{\circ} \mathrm{C}$ overnight, followed by an Alexa Fluor 488-conjugated secondary antibody (Jackson ImmunoResearch Laboratories, Inc., West Grove, PA, USA) for $45 \mathrm{~min}$ at $37^{\circ} \mathrm{C}$. The cells were rinsed in PBS between incubation steps and mounted with medium containing 4'-6-diamidino-2-phenylindole (DAPI). Immunofluorescence images were acquired using a fluorescence microscope (Olympus).

Statistical analysis. The Wilcoxon signed rank test was used for comparison of quickscores between paired ESCC and adjacent benign tissue (Fig. 1B). Comparison of Quickscore distribution between benign and ESCC was performed by 

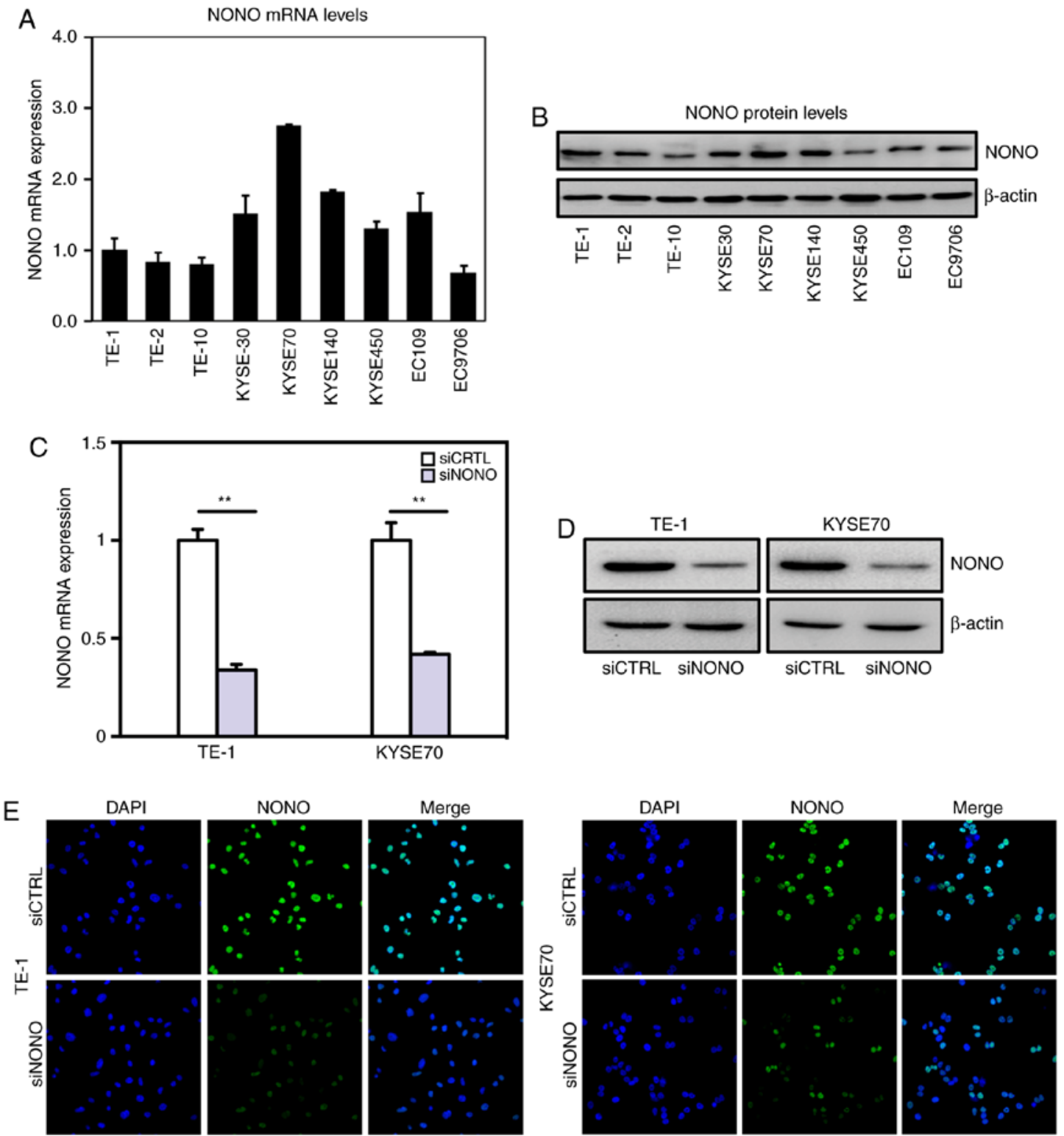

Figure 2. Real-time PCR and western blot analysis of NONO expression in esophageal squamous cell carcinoma (ESCC) cells. (A) Real-time PCR measured relative NONO mRNA levels to GAPDH in 9 ESCC cell lines. (B) Western blot analysis of endogenous expression of NONO in all 9 ESCC cell lines. $\beta$-actin served as a loading control. (C and D) Transfection efficiencies of NONO siRNA knockdown in TE-1, KYSE70 cells were measured by real-time PCR and western blotting. (E) Immunofluorescence staining detected expression and nuclear localization of NONO protein in human ESCC cell lines. NONO expression is reduced after NONO siRNA knockdown in TE-1, KYSE70 cells.

$\chi^{2}$ test (Fig. 1C). The associations of pathological features with NONO quickscores were evaluated by Fisher's exact test in Table I. Data from Figs. 2-4 are presented as the mean \pm SEM that was calculated from at least three independent experiments. Statistical analysis was carried out using GraphPad Prism (version 4) (GraphPad Software, Inc., La Jolla, CA, USA) with the level of significance set at $\mathrm{P}<0.05, \mathrm{P}<0.01$ and $\mathrm{P}<0.001$. Paired Student's t-test was used to compare two groups when the means follow the normal distribution.

\section{Results}

NONO is upregulated in human ESCC tissue samples. Three independent microarray studies reported that higher mRNA levels of NONO were detected in ESCC tissues (Fig. 1A) $(16,17,24)$. By contrast, there were no significant differences of NONO mRNA levels in Barrett's esophagus, EAC and adjacent normal esophagus. In order to determine NONO protein expression in ESCC, we collected 42 paired patient samples containing ESCC and adjacent benign tissue for IHC. IHC signal of NONO was localized in the nuclei of esophageal epithelial cells as well as a sub-group of stromal and muscle cells (Fig. 1B). NONO expression was high in ESCC compared with that in adjacent benign tissue samples $(\mathrm{P}<0.0001)$ (Fig. 1B). Representative IHC images are presented in Fig. 1C. Of the ESCC tissue samples $66.7 \%$ showed strong NONO signal whereas only $11.9 \%$ of benign tissue samples showed strong NONO staining (Fig. 1D). The relationship between pathological background and NONO expression was further analyzed (Table I). While there was no correlation between NONO protein levels with patient age, gender, lymph node metastasis, pathological differentiation and TNM stage, strong 

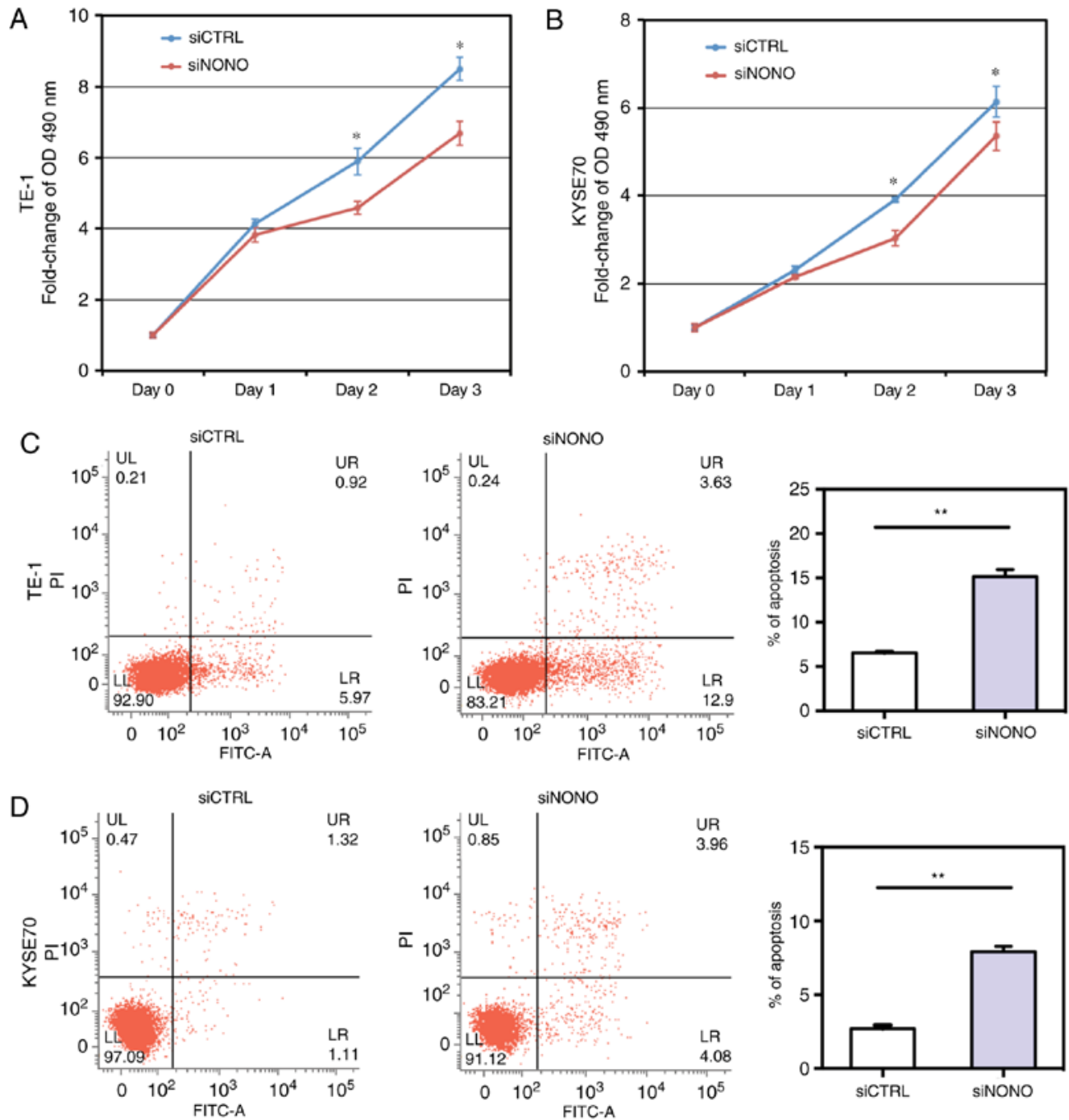

Figure 3. NONO modulates cell proliferation and apoptosis. (A and B) MTS assay showed that NONO knockdown inhibited proliferation in TE-1 and KYSE70 ESCC cell lines. (C and D) Apoptotic cell death was determined by flow cytometric analysis with Annexin V and PI staining. NONO knockdown increased the level of apoptosis in TE-1 and KYSE70 cell lines. Results shown are representative of three independent experiments with Student's t-test. Statistical significance ${ }^{*} \mathrm{P}<0.05$ and ${ }^{* *} \mathrm{P}<0.01$.

A

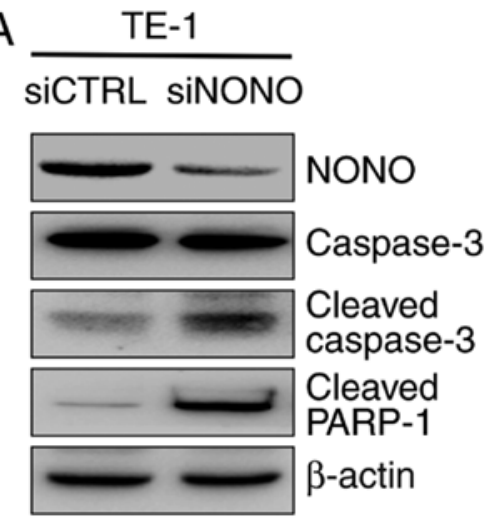

$\mathrm{B}$

$\frac{\text { KYSE70 }}{\text { SiCTRL SINONO }}$

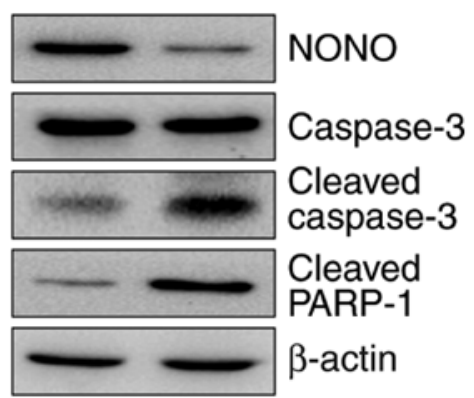

Figure 4. Examination of NONO targets in esophageal squamous cell carcinoma (ESCC) cells. (A) The cleavages of caspase-3, and PARP-1 were compared between siRNA control and siRNA NONO-transfected cells by western blotting at the indicated time-points. $\beta$-actin served as a loading control. NONO depletion upregulated caspase-3 and PARP-1 cleavage in TE-1 and KYSE70 ESCC cell lines.

NONO staining was detected in ESCC with greater tumor invasion depth $(\mathrm{P}=0.011)$. These results suggested a potential role of NONO in ESCC.
NONO expression in ESCC cell lines. Real-time PCR and western blot assays indicated that NONO is widely expressed in multiple ESCC cell lines (Fig. 2A and B). However, NONO 
Table I. Pathological information of ESCC patients and NONO expression.

\begin{tabular}{lcl}
\hline Variables & $\begin{array}{c}\text { NONO expression level } \\
\text { Weak }+ \text { Strong (\%) } \\
\text { moderate }(\%)\end{array}$ & No. P-value \\
\hline
\end{tabular}

Age

$\begin{array}{lrrrr}>60 & 15 & 4(28.6) & 11(39.3) & 0.734 \\ <60 & 27 & 10(71.4) & 17(60.7) & \end{array}$

Sex

$\begin{array}{lrrrr}\text { Male } & 32 & 11(78.6) & 21(75.0) & 1.000 \\ \text { Female } & 10 & 3(21.4) & 7(25.0) & \end{array}$

Tumor depth

$\begin{array}{lrrrrr}\text { T1-2 } & 6 & 5(35.7) & 1(3.6) & 0.011^{\mathrm{a}} \\ \mathrm{T} 3-4 & 36 & 9(64.3) & 27(96.4) & \end{array}$

LN metastasis

$\begin{array}{lrrrr}\text { N0 } & 36 & 11(78.6) & 25(89.3) & 0.652 \\ \text { N1/N2/N3 } & 6 & 3(21.4) & 3(10.7) & \end{array}$

Pathological

differentiation

$\begin{array}{lrrrr}\text { Well } & 7 & 2(14.2) & 5(17.9) & 0.651 \\ \text { Moderate } & 21 & 6(42.9) & 15(53.6) & \\ \text { Poor } & 14 & 6(42.9) & 8(28.5) & \end{array}$

AJCC stage

$\begin{array}{lrrrr}\text { I } & 5 & 2(14.3) & 3(10.7) & 0.860 \\ \text { II } & 26 & 9(64.3) & 17(60.7) & \\ \text { III-IV } & 11 & 3(21.4) & 8(28.6) & \end{array}$

AJCC, American Joint Commission on Cancer. ESCC, esophageal squamous cell carcinoma. The associations of pathological information of ESCC patients with NONO expression are summarized and statistically analyzed by Fisher's exact test. ${ }^{\text {a }}<0.05$, significant difference.

mRNA levels varies among these cell lines with relatively higher levels in KYSE30 and KYSE70 cell lines and lower levels in TE-1, TE-2 and TE-10 cell lines. TE-1 was derived from well differentiated ESCC, whereas KYSE70 was derived from poorly differentiated tumors, respectively (25). We chose TE-1 and KYSE70 cell lines for further functional analyses of NONO. Knockdown NONO was achieved by transient transfection with efficiencies shown in Fig. 2C-E.

Knockdown NONO inhibits ESCC cell proliferation and induces cell apoptosis. Given the elevated expression of NONO in ESCC tissues and cell lines, we examined whether NONO regulated ESCC cell growth. As shown in Fig. 3A and B, NONO knockdown by specific siRNA significantly inhibited growth of TE-1 and KYSE70 cells.

To determine whether NONO could regulate apoptosis of TE-1 and KYSE70 cells, we performed an Annexin V-FITC and PI assay to detect apoptosis. Knockdown of NONO significantly induced apoptosis of TE-1 and KYSE70 cells.
After NONO knockdown, the percentages of both early apoptotic (Annexin V-positive/PI-negative) and late apoptotic (Annexin V-positive/PI-positive) cells were significantly increased in NONO-knockdown cells, compared with control cells $(\mathrm{P}<0.05$, Fig. $3 \mathrm{C}$ and $\mathrm{D})$.

Because apoptosis is often mediated by the activation of caspase-3 that leads to PARP binding to fragmented DNA, western blot analysis was then used to detect caspase-3 activation. The result showed that cleavages of caspase- 3 and PARP-1 were dramatically increased in NONO-knockdown cells, compared with control cells (Fig. 4).

NONO regulates ESCC cell migration and invasion in vitro. Since higher NONO protein expression was observed in tumors with greater local invasion (Table I), we further studied whether NONO is a regulator of ESCC cell migration and invasion in vitro. Knockdown of endogenous NONO expression significantly reduced TE-1 and KYSE70 cell mobility in passing through the surface of culturing plates or penetrating through Matrigel (Fig. 5). These results indicated that the expression levels of NONO are important for ESCC cell migration and invasion.

Knockdown NONO negatively regulates $p$-Erkl/2 and p-AKT expression. As in vitro assays showed that NONO is overexpressed in ESCC tissues and cells, and that knockdown NONO inhibits proliferation, induces apoptosis and reduces mobility of TE-1 and KYSE70 cells, we then explored the molecular mechanism underlying the effects. Both Erk1/2/MAPK and PI3K/AKT activation are frequent events in human cancer including ESCC (26,27). Since Erk1/2 and AKT are activated through phosphorylation, we detected the expression of the Thr202/Tyr204 phosphorylated form of Erk1/2 and Ser473 phosphorylated form of AKT by western blotting. TE-1 and KYSE70 cells were treated with siRNA targeting NONO or control siRNA as a negative. We found that the expression levels of phosphorylated Erk1/2 and AKT were dramatically decreased in both TE-1 and KYSE70 NONO-knockdown cells (Fig. 6). These data suggested that Erk1/2 and PI3K/AKT pathway is required for the NONO-regulated growth, apoptosis and invasion of ESCC cells.

\section{Discussion}

Being one of the most common causes of cancer-related deaths in China, the average 5-year survival rate of ESCC is still low. Several mechanisms promote ESCC progression and provide with independence from normal regulation of cell control (28). Treatment of ESCC especially in advanced stages still remains a clinical challenge. Therefore, new therapeutic targets remain to be elucidated. The nuclear NONO protein is an RNA-binding molecule containing two RNA recognition motifs. It is able to bind double-stranded DNA, single-stranded DNA and RNA (29). Previous studies showed that it retains hyper-edited messenger RNA in the nucleus and therefore influences gene expression and differentiation of stem cells (30). Recently, it has been shown that NONO is strongly expressed in several types of cancer. In addition, an increasing number of studies have reported that NONO modulates various pivotal intermediate molecules and ultimately regulates cell proliferation, apoptosis 
A
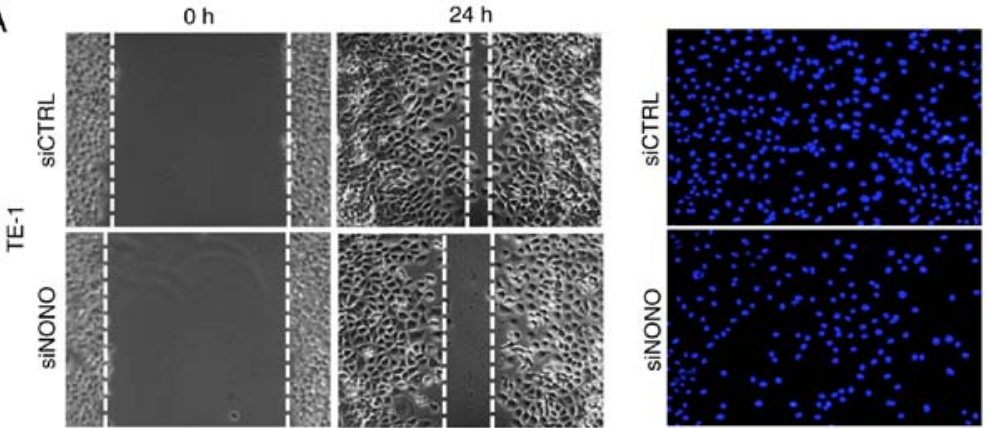

B
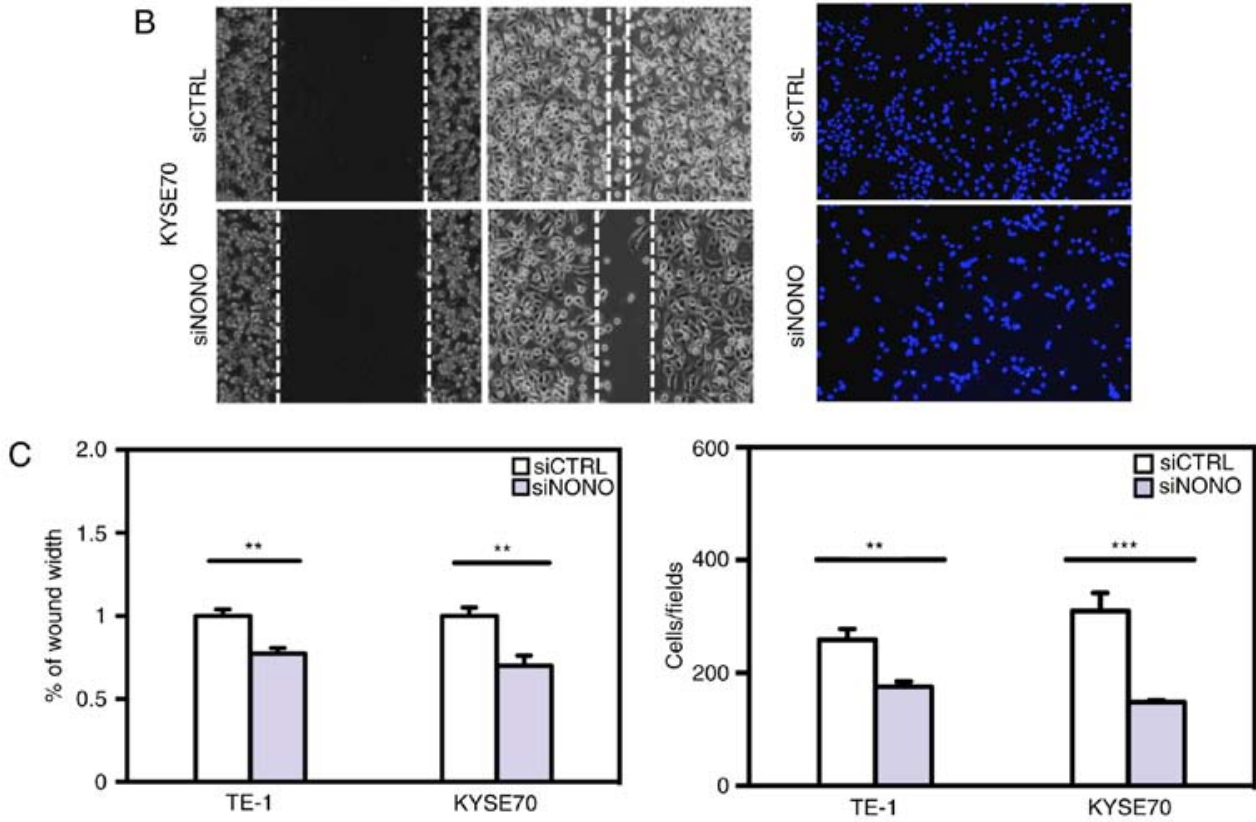

Figure 5. NONO knockdown decreases esophageal squamous cell carcinoma (ESCC) cell migration and Matrigel invasion. TE-1 and KYSE70 cells were transfected with NONO or control siRNA for $24 \mathrm{~h}$. (A and B) Wound healing assay showed that cell motility was inhibited by NONO knockdown. Representative images at time 0 and $24 \mathrm{~h}$ after scratching (left). Cells penetrated through the Matrigel were captured by microscope and representative images are shown (right). The numbers of cells invaded through Matrigel in each condition were counted and plotted. (C) Results shown are representative of three independent experiments with Student's t-test, the statistical significance was ${ }^{*} \mathrm{P}<0.05,{ }^{* *} \mathrm{P}<0.01$ and ${ }^{* * * *} \mathrm{P}<0.001$.
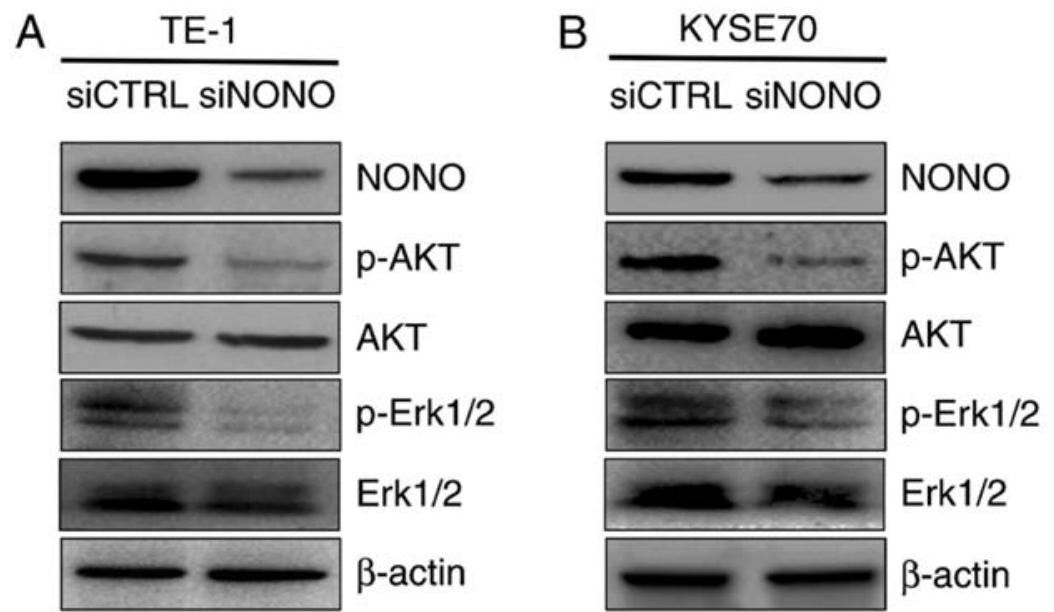

Figure 6. Akt and Erk1/2 are likely the downstream targets of NONO-mediated signaling. p-Akt, Akt, p-Erk1/2 and Erk1/2 were compared between TE-1 and KYSE70 cell lines treated with control siRNA and NONO siRNA by western blot analysis. $\beta$-actin served as a loading control.

and mobility in different types of human carcinomas. However, the expression pattern and function of NONO in the tumorigenesis of ESCC remain unknown. Our contribution in this study is for the first time to demonstrate that NONO protein expression is upregulated in ESCC and that higher NONO protein levels are associated with greater tumor invasion depth. In addition, 
we demonstrated that NONO plays direct regulatory roles in ESCC cell proliferation, apoptosis, migration and invasion involving Erk1/2 and Akt signaling pathways.

Firstly, we observed a significantly elevated NONO expression in most ESCC tissues compared with paired normal esophageal epithelial tissues. Higher NONO expression was shown to be associated with deeper local invasion, but no differences between tumors with different degree of lymph node metastasis, supporting a potential significance in early stage of ESCC progression. However, the correlation of NONO expression and the poor overall survival of ESCC patients need further study.

Secondly, we observed different oncogenic effects in two ESCC cell lines following downregulation of NONO expression. Targeted NONO silencing using siRNA inhibited cell growth in TE-1 and KYSE70 ESCC cell lines. Moreover, NONO silencing increased the percentage of apoptotic cells in those cell lines, in which this oncogene is endogenously overexpressed. In addition, we examined the expression of cleaved caspase- 3 and cleaved PARP-1 by western blotting. We showed that knockdown of NONO could inhibit caspase-3 and PARP-1 cleavage, which was according to its role of apoptosis inhibitor. Taken together, our data suggest that NONO may represent a promising and effective target for antitumor therapy of ESCC.

Recent studies have also shown that the in vitro proliferation, apoptosis and invasion of ESCC cell lines were regulated by inhibition of PI3K or MAPK $(26,31)$. PI3K is a lipid kinase that generates second messengers involved in regulation of a wide spectrum of cellular functions including proliferation, survival and invasion (32). The effects of PI3K on tumor growth and progression are thought to be mediated mainly by Akt. The MAPK family includes the ERKs and the stress-activated protein kinases (SAPKs), p38 and JNK. Erk/MAPK signaling is frequently activated and promotes cancer cell proliferation, cell survival and metastasis $(33,34)$. Some studies observed multiple biological aspects on NONO in tumor cells, such as NONO affecting cell proliferation, apoptosis, and cell cycle of melanomas, which is related to cx-43 (10). Besides, NONO stimulates SREBP-1a-dependent cell proliferation and tumor growth of breast cancer cells in vitro and in vivo (15). This study is the first to report that NONO silencing downregulated the expression level of $\mathrm{p}-\mathrm{Akt}$ and $\mathrm{p}$-Erk1/2 protein without affecting the total Akt and Erk1/2 expression level in ESCC cell lines, which suggested that NONO might play an important role in growth, apoptosis and metastasis of ESCC by activating both the PI3K/ Akt and MAPK/Erk signaling pathways. However, this study was performed only at the cellular level. Further experiments to verify the influence of NONO on ESCC will be done in animal models. More comprehensive investigations are required to determine the associations between NONO, Akt, Erk and tumorigenesis.

Taken together, our results demonstrated that knockdown of NONO could inhibit proliferation and invasion, and promote apoptosis through the PI3K/Akt and MAPK/Erk signaling pathways in ESCC cancer cells. Our data may provide new evidence to improve the understanding of carcinogenesis of ESCC and may help to develop new therapeutic strategies for the treatment of ESCC.

\section{Acknowledgements}

This study was supported by Beijing Municipal Administration of Hospitals Clinical Medicine Development of Special Funding Support (no. ZY201308), and Beijing Natural Science Foundation (no. 7152043).

\section{References}

1. Holmes RS and Vaughan TL: Epidemiology and pathogenesis of esophageal cancer. Semin Radiat Oncol 17: 2-9, 2007.

2. Wang X, Fan JC, Wang AR, Leng Y, Li J, Bao Y, Wang Y, Yang QF and Ren Y: Epidemiology of esophageal cancer in Yanting - regional report of a national screening programme in China. Asian Pac J Cancer Prev 14: 2429-2432, 2013.

3. Jemal A, Siegel R, Xu J and Ward E: Cancer statistics, 2010. CA Cancer J Clin 60: 277-300, 2010.

4. Shav-Tal Y and Zipori D: PSF and p54 (nrb)/NonO - multifunctional nuclear proteins. FEBS Lett 531: 109-114, 2002.

5. Patton JG, Porro EB, Galceran J, Tempst P and Nadal-Ginard B: Cloning and characterization of PSF, a novel pre-mRNA splicing factor. Genes Dev 7: 393-406, 1993.

6. Li S, Kuhne WW, Kulharya A, Hudson FZ, Ha K, Cao Z and Dynan WS: Involvement of p54 (nrb), a PSF partner protein, in DNA double-strand break repair and radioresistance. Nucleic Acids Res 37: 6746-6753, 2009.

7. Dong B, Horowitz DS, Kobayashi R and Krainer AR: Purification and cDNA cloning of HeLa cell p54nrb, a nuclear protein with two RNA recognition motifs and extensive homology to human splicing factor PSF and Drosophila NONA/BJ6. Nucleic Acids Res 21: 4085-4092, 1993.

8. Ito $\mathrm{T}$, Watanabe $\mathrm{H}$, Yamamichi $\mathrm{N}$, Kondo $\mathrm{S}$, Tando $\mathrm{T}$, Haraguchi T, Mizutani T, Sakurai K, Fujita S, Izumi T, et al: Brm transactivates the telomerase reverse transcriptase (TERT) gene and modulates the splicing patterns of its transcripts in concert with p54 (nrb). Biochem J 411: 201-209, 2008.

9. Barboro P, Rubagotti A, Orecchia P, Spina B, Truini M, Repaci E, Carmignani $\mathrm{G}$, Romagnoli A, Introini C, Boccardo F, et al: Differential proteomic analysis of nuclear matrix in muscleinvasive bladder cancer: Potential to improve diagnosis and prognosis. Cell Oncol 30: 13-26, 2008.

10. Schiffner S, Zimara N, Schmid R and Bosserhoff AK: p54nrb is a new regulator of progression of malignant melanoma. Carcinogenesis 32: 1176-1182, 2011.

11. Ishiguro H, Uemura H, Fujinami K, Ikeda N, Ohta $\mathrm{S}$ and Kubota Y: $55 \mathrm{kDa}$ nuclear matrix protein $(\mathrm{nmt55}) \mathrm{mRNA}$ is expressed in human prostate cancer tissue and is associated with the androgen receptor. Int J Cancer 105: 26-32, 2003.

12. Kim YM, Seo J, Kim YH, Jeong J, Joo HJ, Lee DH, Koh GY and Lee KJ: Systemic analysis of tyrosine phosphorylated proteins in angiopoietin-1 induced signaling pathway of endothelial cells. J Proteome Res 6: 3278-3290, 2007.

13. Clark J, Lu YJ, Sidhar SK, Parker C, Gill S, Smedley D, Hamoudi R, Linehan WM, Shipley J and Cooper CS: Fusion of splicing factor genes PSF and NonO (p54nrb) to the TFE3 gene in papillary renal cell carcinoma. Oncogene 15: 2233-2239, 1997.

14. Liu PY, Erriquez D, Marshall GM, Tee AE, Polly P, Wong M, Liu B, Bell JL, Zhang XD, Milazzo G, et al: Effects of a novel long noncoding RNA, IncUSMycN, on N-Myc expression and neuroblastoma progression. J Natl Cancer Inst 106: 106, 2014.

15. Zhu Z, Zhao X, Zhao L, Yang H, Liu L, Li J, Wu J, Yang F, Huang G and Liu J: p54 (nrb)/NONO regulates lipid metabolism and breast cancer growth through SREBP-1A. Oncogene 35: 1399-1410, 2016.

16. Hu N, Clifford RJ, Yang HH, Wang C, Goldstein AM, Ding T, Taylor PR and Lee MP: Genome wide analysis of DNA copy number neutral loss of heterozygosity $(\mathrm{CNNLOH})$ and its relation to gene expression in esophageal squamous cell carcinoma. BMC Genomics 11: 576, 2010.

17. Su H, Hu N, Yang HH, Wang C, Takikita M, Wang QH, Giffen C, Clifford R, Hewitt SM, Shou JZ, et al: Global gene expression profiling and validation in esophageal squamous cell carcinoma and its association with clinical phenotypes. Clin Cancer Res 17: 2955-2966, 2011.

18. Edge SBBD, Byrd DR, Compton CC, Fritz AG, Greene FL and Trotti A (eds): American Joint Committee on Cancer Staging Manual. 7th editon. Springer, New York, NY, 2009. 
19. Sobin LH and Compton CC: TNM seventh edition: what's new, what's changed: communication from the International Union Against Cancer and the American Joint Committee on Cancer. Cancer 116: 5336-5339, 2010

20. Sun X, Qiu JJ, Zhu S, Cao B, Sun L, Li S, Li P, Zhang S and Dong S: Oncogenic features of PHF8 histone demethylase in esophageal squamous cell carcinoma. PLoS One 8: e77353, 2013

21. Meng XY, Zhu ST, Zhou QZ, Li P, Wang YJ and Zhang ST: Promoter methylation regulates cigarette smoke-stimulated cyclooxygenase-2 expression in esophageal squamous cell carcinoma. J Dig Dis 13: 208-213, 2012.

22. Detre S, Saclani Jotti G and Dowsett M: A 'quickscore' method for immunohistochemical semiquantitation: Validation for oestrogen receptor in breast carcinomas. J Clin Pathol 48: 876-878, 1995

23. Zhang N, Sun X, Sun M, Zhu S, Wang L, Ma D, Wang Y, Zhang S and Li P: 4-(Methylnitrosamino)-1-(3-pyridyl)-1-butanone promotes esophageal squamous cell carcinoma growth via betaadrenoceptors in vitro and in vivo. PLoS One 10: e0118845, 2015

24. Kimchi ET, Posner MC, Park JO, Darga TE, Kocherginsky M, Karrison T, Hart J, Smith KD, Mezhir JJ, Weichselbaum RR, et al: Progression of Barrett's metaplasia to adenocarcinoma is associated with the suppression of the transcriptional programs of epidermal differentiation. Cancer Res 65: 3146-3154, 2005.

25. Nishihira T, Hashimoto Y, Katayama M, Mori S and Kuroki T: Molecular and cellular features of esophageal cancer cells. J Cancer Res Clin Oncol 119: 441-449, 1993.

26. Liu F, Zheng S, Liu T, Liu Q, Liang M, Li X, Sheyhidin I, Lu X and Liu W: MicroRNA-21 promotes the proliferation and inhibits apoptosis in Eca109 via activating ERK1/2/MAPK pathway. Mol Cell Biochem 381: 115-125, 2013.
27. Grille SJ, Bellacosa A, Upson J, Klein-Szanto AJ, van Roy F, Lee-Kwon W, Donowitz M, Tsichlis PN and Larue L: The protein kinase Akt induces epithelial mesenchymal transition and promotes enhanced motility and invasiveness of squamous cell carcinoma lines. Cancer Res 63: 2172-2178, 2003.

28. Hanahan D and Weinberg RA: Hallmarks of cancer: The next generation. Cell 144: 646-674, 2011.

29. Basu A, Dong B, Krainer AR and Howe CC: The intracisternal A-particle proximal enhancer-binding protein activates transcription and is identical to the RNA- and DNA-binding protein p54nrb/NonO. Mol Cell Biol 17: 677-686, 1997.

30. Bond CS and Fox AH: Paraspeckles: Nuclear bodies built on long noncoding RNA. J Cell Biol 186: 637-644, 2009.

31. Li B, Tsao SW, Li YY, Wang X, Ling MT, Wong YC, He QY and Cheung AL: Id-1 promotes tumorigenicity and metastasis of human esophageal cancer cells through activation of PI3K/AKT signaling pathway. Int J Cancer 125: 2576-2585, 2009.

32. Fresno Vara JA, Casado E, de Castro J, Cejas P, Belda-Iniesta C and González-Barón M: PI3K/Akt signalling pathway and cancer. Cancer Treat Rev 30: 193-204, 2004.

33. Wada T and Penninger JM: Mitogen-activated protein kinases in apoptosis regulation. Oncogene 23: 2838-2849, 2004.

34. Chan-Hui PY and Weaver R: Human mitogen-activated protein kinase kinase kinase mediates the stress-induced activation of mitogen-activated protein kinase cascades. Biochem J 336: 599-609, 1998

This work is licensed under a Creative Commons Attribution-NonCommercial-NoDerivatives 4.0 International (CC BY-NC-ND 4.0) License. 